\title{
Twin Band-Notched Ultrawideband MIMO Antenna
}

\section{R. Sambasiva Nayak, P. Karpagavalli}

\begin{abstract}
In this article, we have presented various techniques that are used for improving different parameters related to UWB antenna. In this Paper, we planned for MIMO antennas in contemporary wireless communication which enhances the bandwidth and gives compact antennas. The antenna band we notched is of planned MIMO which offers an bandwidth with the operational band-notched. The bandwidth capacity of the antenna is from 2.93-20 gigahertz with sharp rejection at WLAN-band with isolation of not exactly - $22 \mathrm{~dB}$ is accomplished for the whole band, by utilizing a simple modified shaped structure in the bottom plane, port isolation and transmission capacity are improved. The diversity execution performance is likewise contemplated and whole outcomes shows it's a potential point of using MIMO based diversity antenna for ultra wide band applications which is demonstrate in this paper. The parameters to assess the performance of the MIMO are explained, the whole examination completed in different sections has been outlined.
\end{abstract}

Keywords: DGS, UWB, SRR and MIMO

\section{INTRODUCTION:}

MIMO/Diversity strategies impressively improve the reliableness and transmission ability of a framework over single-receiving antenna frameworks while not expanding the data transfers capacity and power utilization. Regardless of various advantages of UWB frameworks flag weakening in multipath conditions is an issue. This disadvantage is settled by joining UWB and MIMO procedures. Utilization of MIMO innovation in ultra-wideband (UWB) frameworks are examined in that demonstrated that it gives a noteworthy information rate improvement over MIMO innovation used in narrowband frameworks like for UMTS, and remote neighborhood. Various procedures are examined to curtail shared coupling between the unique segments in UWB MIMO frameworks. In MIMO antennas were concentrated to support segregation by utilizing various DGS or by presenting stubs and spaces between the 2 different parts. In ACS bolstered with an I-formed concentrate the radiator and by appending an elongated fix on the back, while in a mushroom kind attractive power band hole structure is utilized between 2antennas to broaden the confinement.

EMI is another disadvantage of MIMO gadgets operational in UWB band, a reasonable response to the present downside is to style UWB reception apparatus with band-scored qualities.

Revised Manuscript Received on December 30, 2019.

* Correspondence Author

R. Samba Siva Nayak, Department of ECE, Sri Satya Sai University of Technology and Medical Science, Sehore, Bhopal, Madhya Pradesh, India, sambanayak@gmail.com

Dr P. Karpagavalli, Department of ECE, Sri Satya Sai University of Technology and Medical Science, Sehore, Bhopal, Madhya Pradesh, India

(C) The Authors. Published by Blue Eyes Intelligence Engineering and Sciences Publication (BEIESP). This is an open access article under the CC BY-NC-ND license (http://creativecommons.org/licenses/by-nc-nd/4.0/)
Hence, very surprising methods region unit as indicated by inside the writing to smother impedance like embeddings short stub, a bend molded opening,by carving 2 split ring resonator spaces within the receiving antenna part, and so on. A parasitic T-formed strip is acquainted between the receiving antenna parts with cut back the common coupling and a consolidate of L-molded cuts was engraved on the base to get a notched band. In 2circular shaped different segments bolstered by CPWs territory unit intended to get UWB attributes. By carving, SRR and by the cooperation of the circular segment formed strips and projected stub notched frequencies in band-X with band of remote neighborhood was accomplished. The UWB MIMO reception apparatus in utilized 2 heptagonal monopole parts set symmetrically on the substrate for all time segregation between the 2 input ports, space was cut on everything about receiving antenna segments to make an indent inside the band of remote neighborhood. Within an abandoned ground in simple structure of Y-formed band is recorded inside the ground plane to stifle common coupling 6-between 2 receiving wires and improve the electric obstruction coordinating. Double notched groups zone unit accomplished by carving an open-finished space and a split ring resonator inside the ground plane.

The structured receiving antenna offered UWB transmission capacity from 2.93 to 20 gigahertz and - 22dB disengagement. Be that as it may, a few MIMO antennas with/without band-scored attributes are referenced over, recorded in Table 1 have a nearly bigger size and poor confinement when contrasted with the arranged structure/style. The structured antenna contains a reduced size with low common coupling and in this way gives a reasonable response to a few moveable remote applications. In this segment, a decreased encouraged minimal twin band-notched MIMO/diversity variety opening reception apparatus for UWB applications is arranged. The structured antenna contains a minimized size of $18 \times 34 \mathrm{~mm}$. It comprises of a polygon-formed radiator with $2 \mathrm{~L}$-molded cuts as scored channel structures at a remote neighborhood. An execution of reception apparatus for each by reenactment and analysis demonstrates that the arranged MIMO antenna has reasonable electric obstruction coordinating, low common coupling, and reasonably decent variety execution, all through the UWB band with band-notched attributes at WLAN-band $\quad(5.09 \quad \mathrm{GHz}-5.8 \mathrm{GHz}) \quad$ \& $\quad$ EEE INSAT/Super-Extended C-band (6.3GHz- 7.27 GHz). 
Table 1 analyzes the better execution of the planned antenna as for different reception apparatuses in literature

\begin{tabular}{|c|c|c|c|c|}
\hline Size $(\mathrm{mm} \times \mathrm{mm})$ & ${ }^{s 11}(\mathrm{GHz})$ & Isolation(dB) & Notched-Band [GHz] & Gain \\
\hline $35 \times 40=1400$ & $3.1 \cdot 10.6$ & -16 & - & within $3.1 \mathrm{~dB}$ \\
\hline $26 \times 40=1040$ & $3.1 \cdot 10.6$ & -15 & - & 0.9 to $6.5 \mathrm{~dB}$ \\
\hline $30 \times 40=1200$ & $3-10.6$ & -16 & - & - \\
\hline $26 \times 26=676$ & $3.1-10.6$ & -15 & - & $0.85 \mathrm{AB}$ \\
\hline $50 \times 50=100$ & $2.76-10.75$ & -15 & $4.75-6.12$ & $28 \mathrm{~dB}$ \\
\hline $48 \times 48=2304$ & $25-12$ & -18 & $5.1-6.0$ & stable $3 \mathrm{~dB}$ \\
\hline $38.5 \times 38.5=1482.25$ & $3.08-11.8$ & -15 & 5.03-5.97 & 1.4 to $3.6 \mathrm{~dB}$ \\
\hline $30 \times 30=900$ & $3.5-10.6$ & -15 & $5.1-5.8 \& 7.9-8.4$ & - \\
\hline $18 \times 34=612$ & $2.9-20$ & -22 & {$[5.1-5.8) \approx(6.7-7.1]$} & 0 to $7 \mathrm{AB}$ \\
\hline
\end{tabular}

\section{METHODOLOGY:}

As given in first figure the receiving wire geometry of dissimilar segments with a standard ground plane. The general elements of the arranged reception apparatus territory unit exclusively $18 \times 34 \mathrm{~mm}^{2}=612 \mathrm{~mm}^{2}$ or in regards to $0.18 \lambda_{0} \times 0.34 \lambda_{0}$ here free space medium is loud recurrence $3.0 \mathrm{GHz}$. In any case, for getting the lower values of cutoff ultra wide band apparatus of reception is the minimum need recurrence for example 3.1 gigahertz though keeping up the conservativeness of the look. Fundamental plan/style of the reception apparatus begins with picking the receiving antenna structure and its measurements to fulfill the operational recurrence needs.

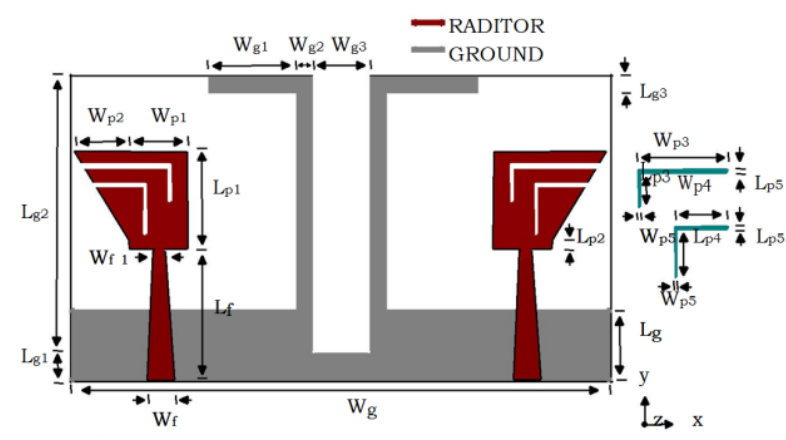

Figure1. Proposed Structure with Dimensions

Monopole association is picked for a scaled down structure/style of UWB antenna and furthermore the essential lower resounding recurrence of an arranged monopole might be approximated by the consequent condition. The arranged radiator is implied with a blend of rectangular $(\mathrm{Lp} 1 \times \mathrm{Wp} 1)$ and triangular (height 4 millimeter and base $5.15 \mathrm{~mm}$ ) stubs to make an exceptional two-dimensional figure shape. With a micro strip of decreased size Lf $\times$ Wf that is placd at each radiator lower edges, we bolstered the 2-D molded figure radiator. The 2 frames such as rectangular and $\mathrm{T}$ frames which is in arranged antennas base plane stubs. Further, which is changed to drawing an elongated molded opening to make a remarkable reversed L-formed ground plane to help the detachment between 2 reception apparatuses as appeared in Figure 1. The arranged MIMO reception apparatus is designed with the substrate of covered FR4 machine of MITS Eleven research center PCB (width $=1.6 \mathrm{~mm}, \rho_{\mathrm{r}}=4.5 \&$ protector misfortune digression is 0.03 . for developing a definitive plan/style zone unit appeared in Figure 2. We MIMO antenna is arranged as, have 2 indistinguishable represented with wavelength $\lambda_{0}$ in predetermined beginning

entirely depends on geometries which are unexpected.
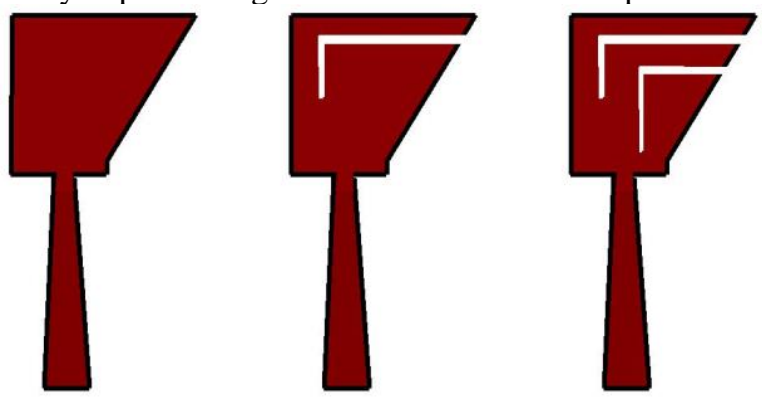

Figure2. Structure of Radiator A, Radiator B, and Radiator A

We use the L- form ground plane on single feature and the radiator of polygon-molded on other feature of antenna may reduces the sustainability line on the contrary aspect of the substrate zone unit got ready for UWB execution. In the way of accomplishing UWB execution, the receiving antenna is increasingly changed Antenna B to stifle impedance at remote neighborhood band. A simple L-molded cut is recorded inside the higher part of the radiator to smother the remote neighborhood band $(5.09-5.8 \mathrm{GHz})$ in UWB band has appeared in Figure3., an L-formed cut (see Antenna C) is engraved inside the lower part of the radiator as appeared in Figure 2. The mimicked S11 for every one of the geometries used in the advancement of a definitive plan/style appears in Figure 3.

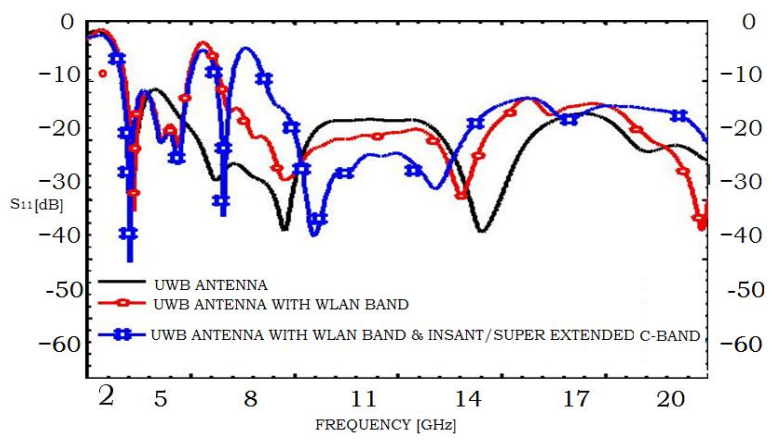

Figure3. S11 of Radiator A, Radiator B, and Radiator C

The determined recurrence $f_{r}$ which is near to value of 4.2 $\mathrm{GHz}$ reenacted lead to Figure 4.

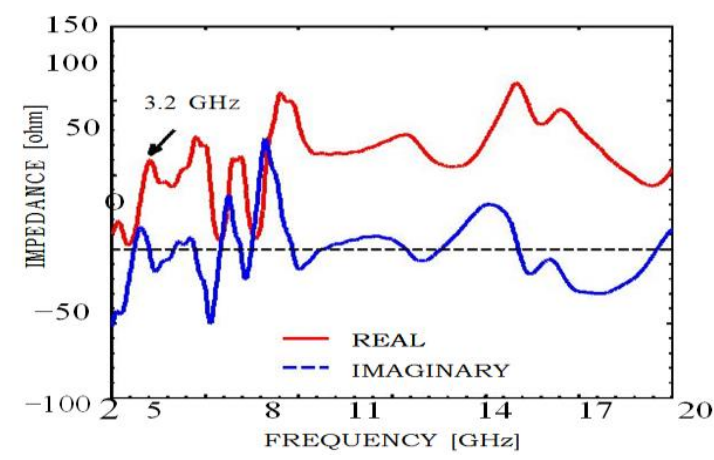

Figure4. Simulated Input electric opposition versus Frequency for the arranged UWB MIMO Antenna appeared in Figure 1

Published By:
Blue Eyes Intelligence Engineering \& Sciences Publication 


\section{RESULT OF GND PLANE:}

The base plane assumes a noteworthy job in the execution of the arranged antenna. It, not just records for higher electrical marvel coordinating of the antenna segments anyway furthermore to upgrade the seclusion between them.

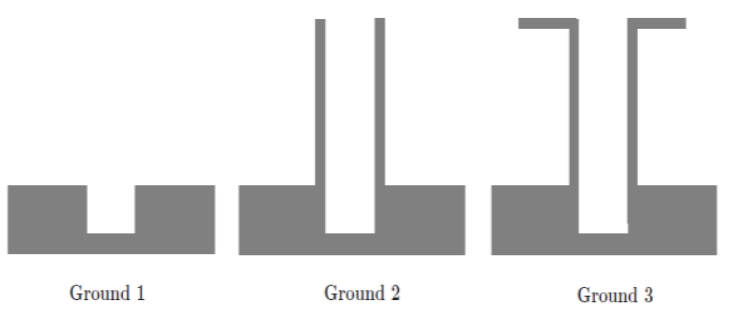

Figure5. Different Ground Structures in new a Final Structure Ground C

Figure5 demonstrates the receiving wire venture of advancement base plane of arranged MIMO. That every part of antenna receiving type region unit indistinguishable, the S11 and S12 will be somewhat similar to S22 and S21 as represented in Figure 6a and 6b. Be that as it may, tfor having the movement of reverberation in the ground plane of 3.7GHz and with the two strips of rectangular size are placed, be that as it may, the common coupling for Ground two inside the recurrence band underneath 4 gigahertz is incredibly poor as appeared in Fig. 6b. Further, by utilizing upset L-molded strips inside the ground plane as appeared in Ground three, the let reverberation recurrence moves down to 3.2 gigahertz with a lower cut off recurrence at $2.9 \mathrm{GHz}$.

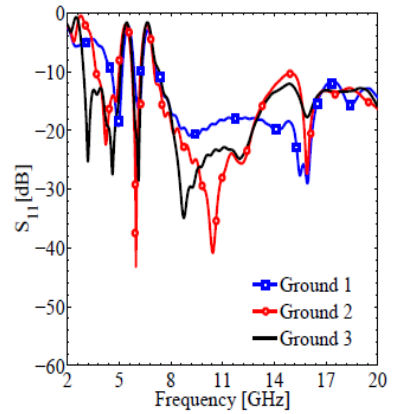

(a)

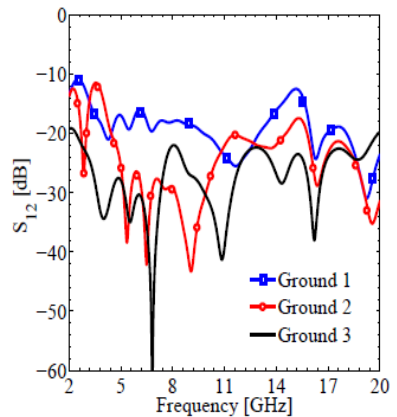

(b)
Figure6. Reenacted S-parameters against Frequency for the differed Ground arrangements (a) S 11 and (b) S12

Segregation in under - twenty decibels acquired that was enough sweet for MIMO antenna execution which is low significantly.

\section{DISTRIBUTION:}

as the structure of decoupling the adequacy is there in the ground lane as shown in figure 7 a-c. For having current adequacy while port one in the ground plane is done then port two is operated similarly when port 2 is done port one is operated as in figure 7(a) which is plainly observed in Lplanes as one begins other ends. As we are familiar that port 1 is having current on the ground plane then after ending the other port is started in the base plane with I-cuts animation which is shown in Figure 7 (b), for example, diminishes control result port one to port two anyway some bit of the present keeps on being connected to second port that progressively gives weak disengagement.

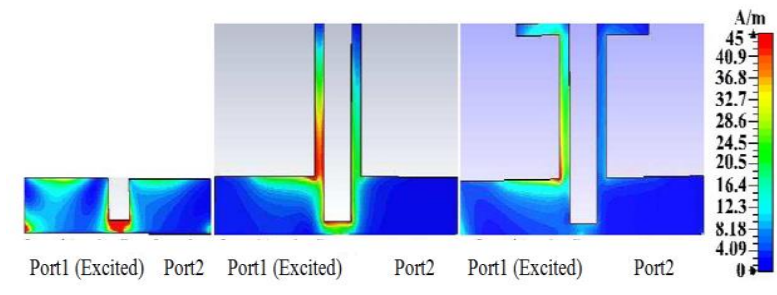

a

Figure7. Geometry of plane ground bottom

L- strip is transformed further form I- strip which is shown in Figure 7(c) incredibly amassed (S12 and S21 region unit over - 20 decibels all through the total band of operational) as shown in figure $6 \mathrm{~b}$ the gaps among the two ports 1 and 2 .

\section{RESULTS:}

Agilent (N5230A) vector organize analyzer is utilized to approve the mimicked outcomes acquired for the arranged MIMO reception apparatus. Mimicked and estimated consequences of S11/S12 and S21/S22 can be seen in Fig 8. The arranged antenna offers electrical wonder transfer speed of 17.07 gigahertz from (2.93 to $20.0 \mathrm{GHz}$ ). The separation between 2 radiators is higher than - 22 decibels over the total working band. The arranged reception apparatus indicates double band indent qualities to smother impedance. As middle recurrence for WLAN and C-band contains an estimation of S11 $=-1.5$ decibel that is sufficiently high to demonstrate viable concealment. The deliberate outcomes are in reasonable concurrence with reproduced results.

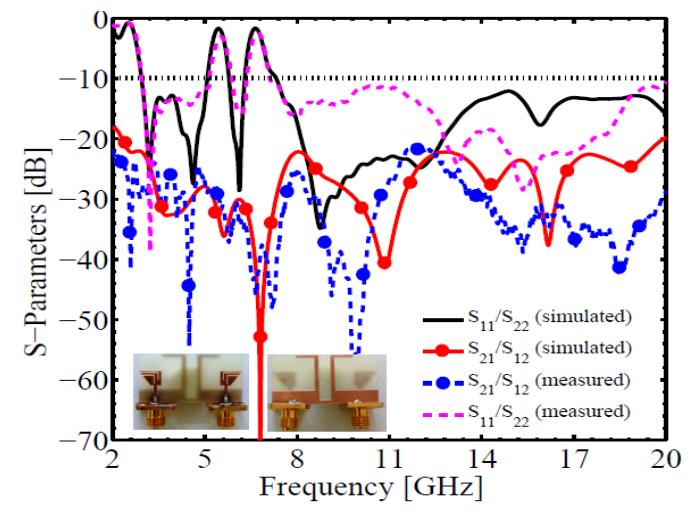

Figure8. Simulated and Measured S11 and S12 -parameters of the proposed MIMO antenna

Patterns: diagram 9 demonstrates the 2-D radiation designs as shown in figures a, b, c, d, e, $\mathrm{f}$ for the arranged MIMO antenna, in the $\mathrm{x}-\mathrm{z}, \mathrm{y}-\mathrm{z}$ and furthermore the $\mathrm{x}-\mathrm{y}$ planes. Over the band which we predefined the antenna posses the radiation pattern of omni directional pattern of the receiving antennas we arranged with furthermore, at the frequencies of band score there is decrease in reception apparatus part of increase.. Additionally, as shown in above diagram demonstrates as radiation designs fall apart at the upper frequencies as a result of the tearing of the radiation lobes. 


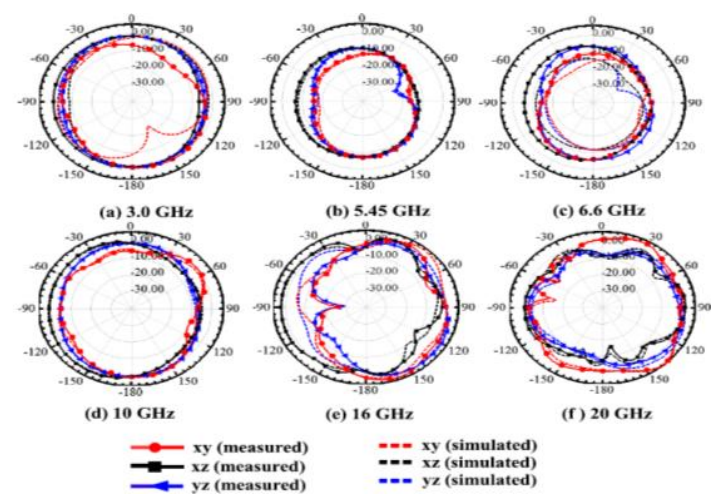

Figure 9. Radiation Pattern at a (A) 3.0 gigahertz (B) 5.45 gigahertz (C) 6.3 gigahertz (D) 8.0 gigahertz (E) 18.0 gigahertz, and (F) 20.0 gigahertz.

Performances: The MIMO conduct of the arranged receiving antenna is assessed as far as ECC, DG and MEG. The relationship among the furthermore segments of nearby placed antenna shared coupling of each amount reception part of coupling is examined further as far as the envelope Connection coefficient; the ECC will be determined misuse S-parameters.

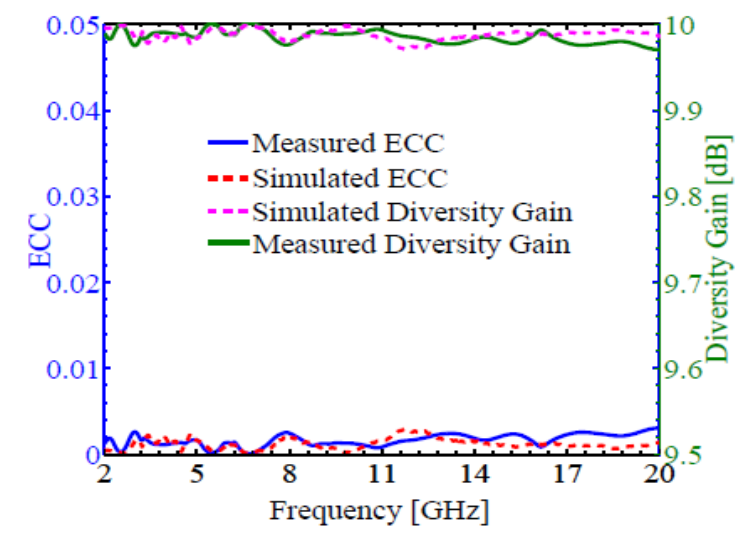

Figure10. Measured and Simulated ECC and DG

The ECC should be in a perfect world be zero for a randomly diversity variety reception apparatus anyway it's down as far as possible was less than 0.4 . The 13th figure demonstrates about reproduced with estimated bends to the antenna we have arranged. The decent variety reception apparatus determined utilizing S-parameter is $<0.01$. as we can see in figure above the ECC with decigram abuse which is found as less than 0.02 in parameter $\mathrm{S}$ and more than $10 \mathrm{~dB}$ in it.

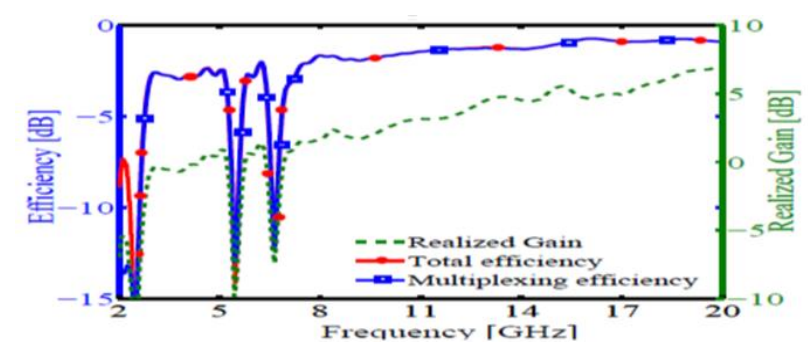

Figure11. Efficiency and DG for the planned MIMO Antenna

Figure 11 shows, the radiation proficiency, and multiplexing productivity and achieved addition to the Multiple Input and Multiple Output type antenna. A productivity multiplexing is characterizes like a result of a refinement inside the power required for a MIMO (Antenna underneath Test) to get a given capacity when contrasted with perfect reference MIMO antenna. It's highly indistinguishable that single port efficiency totally and multiport multiplexing proficiency which is a clear cut picture in figure 11. As a practical parameter the productivity of multiplexed data is professed for information rate up gradation, that not exclusively represents the entire receiving antenna effectiveness, anyway furthermore for connection and proficiency awkwardness. At 5.45 gigahertz and $6.6 \mathrm{GHz}$, there's an impressive drop in reception apparatus effectiveness and this profound dropped by proficiency enables the receiving antenna to figure in high impedance environment. the general increase over the operational band ranges from zero to seven decibel, at score groups the addition drops to - 10 decibel $(5.45 \mathrm{GHz})$ and -8 decibels $(6.6 \mathrm{GHz})$. To have an characterized channel and diverse execution of diverse verity of channel executed for decently, the ratio of large integer of the 2 antenna components ought to full-fill the standards $|\mathrm{MEGi} / \mathrm{MEGj}|<$ $\pm 3 \mathrm{~dB}$.

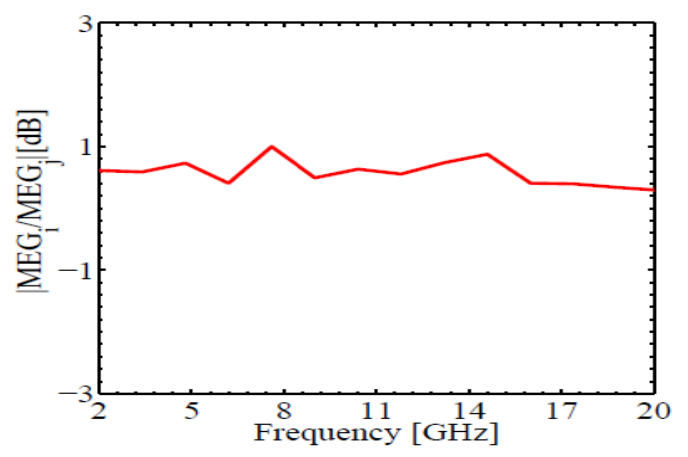

Figure12. Quantitative Relation of MEGs of 2 Antenna segments

From Figure 12 it's found that for the arranged MIMO receiving antenna the quantitative connection of huge whole number lays in the middle for whole band of Ultra Wide Band frequency in the small extent which is named above in total, with a vast majority as one decibel in $6.5 \mathrm{GHz}$.

To the multi-port reception apparatus frameworks, contiguous receiving antenna segments affect each other and once operational in the meantime they affect the general operational data transmission and effectiveness. The fresh out of the box new measurement that is meant on the grounds that the TARC has been acquainted with require this outcome into thought. It will be sketched out on the grounds that the foundation of the quantitative connection of absolute reflected capacity to the frame work of general Multiple Input and Multiple Output antenna type for power evidence which shows the losses in the while antenna. To have the dual access MIMO framework, we can explain it with an resulting condition. A TARC worth of $\angle 0 \mathrm{~dB}$ is intriguing for a MIMO framework. The deliberate and reenacted estimations of TARC are a unit appeared in Figure 4.16. It's found from Figure 16 that the value of TARC for the arranged reception apparatus is a little sum than - 20 decibel for the total band. 


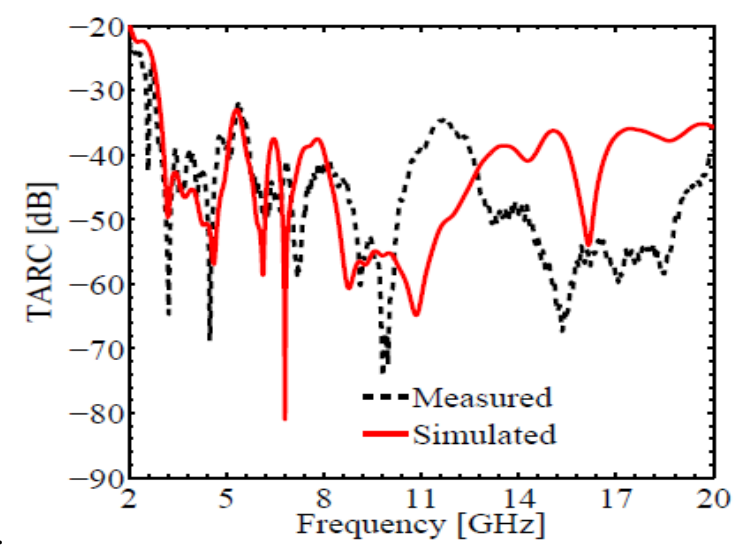

Figure.13. TARC versus Frequency

\section{CONCLUSIONS:}

This experiment gives an Improved UWB/MIMO antenna with dual-band notched characteristics is arranged. The bandwidth capacity of the antenna is from 2.93-20 gigahertz and IEEE INSAT/Super-Extended C-band (6.3-7.27 GHz) with isolation of not exactly - $22 \mathrm{~dB}$ is accomplished for the whole band, by utilizing bottom planes structure of L-shaped which is modified simply, the capacity of isolation port and transmission are optimized. Similarly the performance of diverse execution and every outcome explains that MIMO is a contender of potential and is contemplated for compact UWB applications. In the wake of planning MIMO/ diversity antennas, the whole examination completed in different sections has been outlined.

\section{REFERENCES:}

1. Kang, Gong, "Compact broadband printed slot-monopole-hybrid diversity antenna for mobile terminals," IEEE Antenna and Wireless Propagation Letters, vol. 10, pp. 159-162, 2011.

2. I. Yuk, "Compact MIMO antenna for portable devices in UWB applications," IEEE Transactions on Antennas and Propagation, pp. 4257-4264, August 2013.

3. Mohammad, Ali nezhad "A dual-band WLAN/UWB printed wide slot antenna for MIMO/diversity applications," Microwave and Optical Technology Letters, vol. 55, pp. 461-465, March 2013.

4. Sonkki and E. Salonen, "Low mutual coupling between monopole antennas by using two slots," IEEE Antenna and Wireless Propagation Letters, vol. 9, pp. 138-141, 2010.

5. Crespo, and C. Ling, "UWB portable prin ted monopole array design for MIMO communications," Microwave and Optical Technology Letters, vol. 52, pp. 889- 895, April 2010.

6. K. Mishra, and J. Mukherjee, "A compact dual-band fork- shaped monopole antenna for Bluetooth and UWB applications," IEEE antenna and wireless propagation letters, vol. 10, pp. 627-630, 2011.

7. P. Tran and A. Sibille, "Spatial multiplexing in UWB MIMO communications," Elec-tronics letters, pp. 931-932, August 2006.

8. Chandel, Gautam and Binod Kr. Kanaujia, "A Compact Rhombus-shaped Slot Antenna Fed by Microstrip-line for UWB Applications," International Journal of Microwave and Wireless Technologies, pp. 1-7, 2015.

9. N. Alsath and M. Kanagasabai, "Compact UWB monopo le antenna for automotive communications," IEEE Transactions on Antennas and Propagation, vol. 63, pp. 4204-4208, Sep 2015.

\section{AUTHOR'S PROFILE}

R. Sambasiva Nayak is a Research Scholar in the Department of ECE at Sri Satya Sai University of Technology and Medical Sciences, Sehore, Madhya Pradesh. He has completed Graduated in Electronics and communication engineering from Acharya Nagarjuna University, India and Post Graduated in Digital Electronics and Communication Systems from Jawaharlal Nehru Technological University Hyderabad, India. He has 15 years of teaching, research, and administrative experience. He has been active in research for more than 10 years and 120 papers published in
National Conferences, International Conferences, and National/International reputed and indexed Journals, Scopus journals and including SCI journals in the field of Communications. He is a Life Member in Indian Society for Technical Education, International Association of Engineers, International Association of Computer Science and Information Technology, Universal Association of Computer and Electronics Engineers \& Editorial Board Member. His research interests Antennas, Mobile/Cellular Systems, and Digital Image Processing.

Dr P.Karpagavalli has more than 21 years of teaching, research, and administrative experience. She has published more than 50 papers in National Conferences, International Conferences, and National/International reputed and indexed Journals, Scopus journals and including SCI journals in the field of Communications. She has been actively involved in research and guiding students in the area of Antennas, Signal Processing, VLSI and implementation of Speech CODECs. Currently, she has been working as Associate Professor in ECE, SSSUTMS. 